\title{
Correction to: Effect of selenium supplementation on antioxidant markers: a systematic review and meta-analysis of randomized controlled trials
}

\author{
Motahareh Hasani ${ }^{1} \cdot$ Shirin Djalalinia ${ }^{2,3} \cdot$ Maryam Khazdooz $^{1} \cdot$ Hamid Asayesh $^{4} \cdot$ Maryam Zarei $^{5}$. \\ Armita Mahdavi Gorabi ${ }^{6} \cdot$ Hossein Ansari $^{7}$ - Mostafa Qorbani ${ }^{8,9} \cdot$ Ramin Heshmat $^{10}$
}

Published online: 1 July 2020

(C) Hellenic Endocrine Society 2020

\section{Correction to: Hormones (2019) 18:451-462 https://doi.org/10.1007/s42000-019-00143-3}

The original version of this article, published on 10 December 2019 contained a mistake. One of the affiliations was incorrect. The correct affiliation of Hossein Ansari is given below. Department of Epidemiology and Biostatistics, Health Promotion Research Center, Zahedan University of Medical Sciences, Zahedan, Iran

Publisher's note Springer Nature remains neutral with regard to jurisdictional claims in published maps and institutional affiliations.

The online version of the original article can be found at https://doi.org/ 10.1007/s42000-019-00143-3

Mostafa Qorbani

qorbani9@yahoo.com

$\triangle$ Ramin Heshmat

rheshmat@tums.ac.ir

1 School of Public Health, Iran University of Medical Sciences, Tehran, Iran

2 Development of Research \& Technology Center, Deputy of Research and Technology, Ministry of Health and Medical Education, Tehran, Iran

3 Non-Communicable Diseases Research Center, Endocrinology and Metabolism Population Sciences Institute, Tehran University of Medical Sciences, Tehran, Iran

4 Department of Medical Emergencies, Qom University of Medical Sciences, Qom, Iran
5 Department of Nutrition and Dietetics, Faculty of Medicine and Health Sciences, Universiti Putra Malaysia, Serdang, Selangor, Malaysia

6 Department of Basic and Clinical Research, Tehran Heart Center, Tehran University of Medical Sciences, Tehran, Iran

7 Department of Epidemiology and Biostatistics, Health Promotion Research Center, Zahedan University of Medical Sciences, Zahedan, Iran

8 Non-Communicable Diseases Research Center, Alborz University of Medical Sciences, Karaj, Iran

9 Endocrinology and Metabolism Research Center, Endocrinology and Metabolism Clinical Sciences Institute, Tehran University of Medical Sciences, Tehran, Iran

10 Chronic Diseases Research Center, Endocrinology and Metabolism Population Sciences Institute, Tehran University of Medical Sciences, Tehran, Iran 